\title{
On Context-Tree Prediction of Individual Sequences
}

\author{
Jacob Ziv and Neri Merhav \\ Department of Electrical Engineering \\ Technion - Israel Institute of Technology \\ Haifa 32000, ISRAEL \\ [jz, merhav] @ee.technion.ac.il
}

July 14, 2005

\begin{abstract}
Motivated by the evident success of context-tree based methods in lossless data compression, we explore, in this paper, methods of the same spirit in universal prediction of individual sequences. By context-tree prediction, we refer to a family of prediction schemes, where at each time instant $t$, after having observed all outcomes of the data sequence $x_{1}, \ldots, x_{t-1}$, but not yet $x_{t}$, the prediction is based on a "context" (or a state) that consists of the $k$ most recent past outcomes $x_{t-k}, \ldots, x_{t-1}$, where the choice of $k$ may depend on the contents of a possibly longer, though limited, portion of the observed past, $x_{t-k_{\max }}, \ldots, x_{t-1}$. This is different from the study reported in [1], where general finite-state predictors as well as "Markov" (finite-memory) predictors of fixed order, where studied in the regime of individual sequences.

Another important difference between this study and [1] is the asymptotic regime. While in [1], the resources of the predictor (i.e., the number of states or the memory size) were kept fixed regardless of the length $N$ of the data sequence, here we investigate situations where the number of contexts, or states, is allowed to grow concurrently with $N$. We are primarily interested in the following fundamental question: What is the critical growth rate of the number of contexts, below which the performance of the best context-tree predictor is still universally achievable, but above which it is not? We show that this critical growth rate is linear in $N$. In particular, we propose a universal context-tree algorithm that essentially achieves optimum performance as long as the growth rate is sublinear, and show that, on the other hand, this is impossible in the linear case.
\end{abstract}

Index Terms: context-tree algorithm, universal prediction, finite-state machine, finite-memory machine, predictability, individual sequence.

\section{Introduction}

The problem of universal prediction of stochastic processes as well as individual sequences has received considerable attention throughout the years, in the literature pertaining to a large variety of disciplines, such as information theory, statistics, control theory, finance, and others (see [4] for a survey of some of the results on the theoretical aspects). 
In [1], the problem of universal prediction of individual sequences relative to the class of finite-state predictors was investigated. Given an infinitely long binary sequence $\boldsymbol{x}=$ $\left(x_{1}, x_{2}, \ldots\right)$, the finite-state predictability, $\pi(\boldsymbol{x})$, was defined as

$$
\pi(\boldsymbol{x})=\lim _{S \rightarrow \infty} \limsup _{N \rightarrow \infty} \pi_{S}\left(x_{1}, \ldots, x_{N}\right)
$$

where $\pi_{S}\left(x_{1}, \ldots, x_{N}\right)$ is the minimum relative frequency of prediction errors achieved among all finite-state (FS) predictors with no more than $S$ states, when operating on the first $N$ bits, $x_{1}, \ldots, x_{N}$, of the infinite sequence $\boldsymbol{x}$. An FS predictor with $S$ states, or, an $S_{-}$ state predictor for short, is in turn defined by a next-state function $s_{t+1}=g\left(x_{t}, s_{t}\right) \in \mathcal{S}$, $|\mathcal{S}| \leq S$, which recursively updates the state upon receiving a new input, $x_{t}$, and by an output function $\hat{x}_{t+1}=f\left(s_{t}\right)$, which provides the prediction of $x_{t+1}$. The main contribution in [1] was in proposing a universal (randomized) prediction scheme that achieves $\pi(\boldsymbol{x})$ for every $\boldsymbol{x}$. This scheme was based on the incremental parsing procedure of the Lempel-Ziv algorithm [10]. Note that since $\pi(\boldsymbol{x})$ is defined by taking the limit of $S \rightarrow \infty$ after the limit supremum over of $N \rightarrow \infty$, the regime of the asymptotics dictates that $N$ is very large compared to $S$.

The present study differs from [1] in two main aspects. The first is that we confine attention to context-tree prediction, which means that the current state, $s_{t}$, does not necessarily evolve recursively according to a particular next-state function $g$, but may rather correspond to a certain context, that is, a certain portion of the most recent past $\left(x_{t-k}, x_{t-k+1}, \ldots, x_{t-1}\right)$, where $k$ may vary dynamically according to a certain suffix tree, which is subjected to design. The motivation for exploring context-tree strategies stems from their relative simplicity and their success in lossless data compression applications (see, e.g., [3],[5],[6],[7],[8],[9] and references therein). Quite recently, a context-tree approach was analyzed also in universal prediction of stochastic processes under certain regularity conditions [2],[11],[12]. Also, as was shown in [1], the FS predictability is attainable by finite-memory predictors (also referred to as "Markov predictors" therein), where $k$ is fixed, a-fortiori, it is attainable by the more general class of context-tree predictors, where $k$ is allowed to vary.

The second aspect of the difference between this work and [1] is that here we no longer confine ourselves to the regime where $N>>S$. By allowing $S$ to grow with $N$ at a certain rate, the performance analysis pertaining to the relative effectiveness of context-tree 
predictors may become more refined and informative in the sense that it has the potential to reveal their advantage over ordinary finite-memory predictors, which under the regime of [1], are asymptotically as good as general FS predictors anyway, as mentioned above. Contexttree predictors are intuitively superior to finite-memory predictors of fixed order because, as in data compression, they allow the flexiblility to allocate more memory resources (longer contexts) to the "typical" patterns, that occur more often than others, and less resources (shorter contexts) to the non-typical ones.

The question that we pose then is the following: What is the critical growth rate of $S=S_{N}$ as function of $N$, such that below this rate, the asymptotic optimum contexttree prediction performance of every sequence is still universally achievable, but above this rate, it is not? The answer turns out to be that this critical rate is linear in $N$. More precisely, if $S_{N}=a N,(a-$ positive constant), then no universal predictor (deterministic or randomized) can attain the optimum context-tree prediction performance corresponding to $a N$ contexts, simulatenously for all sequences. Furthermore, for $a=1$, it is easy to show that the value of this optimum prediction performance (in terms of the relative error rate) is zero for any sequence. For a sublinear growth rate of $S_{N}$, on the other hand, we propose a universal context-based prediction algorithm, whose number of contexts grows slightly faster than $S_{N}$, and which asymptotically attains the context-tree predictability pertaining to $S_{N}$ states, for every $\left(x_{1}, \ldots, x_{N}\right)$.

The outline of the paper is as follows. In Section 2, we give a formal definition of the problem and state the main result. Sections 3 and 4 are devoted to proofs.

\section{Problem Formulation and Main Result}

Let $x^{N}=\left(x_{1}, x_{2}, \ldots, x_{N}\right), x_{t} \in\{0,1\}, t=1, \ldots, N$, designate a binary data sequence to be sequentially predicted. A context-tree predictor with $S$ contexts (or, with $S$ leaves) is defined as follows. The output function, $f(\cdot)$, of the predictor is given by

$$
\hat{x}_{t+1}=f\left(s_{t}\right)
$$

where $\hat{x}_{t+1} \in\{0,1\}$ is the predicted value for $x_{t+1}$ and $s_{t}$ is the current context (or, state), which takes on values in a finite set $\mathcal{S},|\mathcal{S}| \leq S, S$ being a positive integer. We allow also randomized output functions, namely, random selection of $\hat{x}_{t+1} \in\{0,1\}$ with respect to (w.r.t.) a conditional probability distribution given $s_{t}$. The context $s_{t}$ is determined from 
the past, $\left(\ldots, x_{t-1}, x_{t}\right)$, by the choice of a context tree, which is a complete ${ }^{1}$ binary tree with $S$ leaves. At time $t$, after having observed $x_{t}$, the context $s_{t}$ is determined by reading off the most recent data symbols in reversed order (first $x_{t}$, then $x_{t-1}$, etc.) and traversing along the tree according to these symbols, starting at the root and ending at a leaf, unless the depth of this leaf is larger than $t$ (which may happen at the beginning of the sequence), in which case we stop at $x_{1}$. Denoting the resulting depth by $k=k\left(\ldots, x_{t-1}, x_{t}\right)$, the context will then be given by $s_{t}=\left(x_{t-k+1}, \ldots, x_{t}\right){ }^{2}$ Thus, the context-tree is used as a suffix tree. A context-tree predictor with $S$ contexts is then defined by a combination of a context-tree with a context set $\mathcal{S}$ and an output function $f: \mathcal{S} \rightarrow\{0,1\}$ (or a set of conditional distributions $\{P(\cdot \mid s), s \in \mathcal{S}\}$ in the randomized case). We denote by $\mathcal{P}_{S}$ the class of all context-tree predictors with $S$ contexts.

Let us now expand the class of predictors $\mathcal{P}_{S}$ according to the following model: Given a total budget of $S$ states, we have the freedom to split it into two subsets of states. One subset of states, of size $S^{C} \in\{1,2, \ldots, S\}$, is dedicated to a context-tree of $S^{C}$ leaves, as before (with $S$ being replaced by $S^{C}$ ). The states in this subset will be referred to as context-tree states. The other subset of states, of size $S^{T} \leq S-S^{C}$, is dedicated to a finite-state machine induced by a prefix tree, which is a complete binary tree with a total of $S^{T}$ nodes (including the root and the internal nodes, but not the leaves). The states in this subset will be referred to as transient states, and each one of the $S^{T}$ transient states corresponds to the root or to an internal node in the prefix tree. The system then works as follows: It begins at the subset of transient states, and the initial state, $s_{1}$, is always the root of the prefix tree. As long as $s_{t}$ is an internal node (or the root) of this tree, the next state $s_{t+1}=g\left(x_{t}, s_{t}\right)$ is the child of $s_{t}$ corresponding to the binary value of $x_{t}$, provided that this child is an internal node as well, otherwise (i.e., if this child is a leaf), then the system passes to the subset of context-tree states, and then $s_{t+1}$ will be the context pertaining to time $t+1$. From this point onward, the system remains in the subset of context states, and operates as described in the previous paragraph. Thus, the transient states are used only at the beginning of the sequence, but at certain time $t$ (that may depend on the contents of $\left.\left(x_{1}, \ldots, x_{t}\right)\right)$, there is a transition into the context state set. We refer to these two modes of operation of the system as the transient mode and the context-tree mode, respectively.

\footnotetext{
${ }^{1}$ By complete binary tree, we refer to a binary tree where every node that is not a leaf has two children.

${ }^{2}$ Note that $k$ cannot exceed $S-1$, and so, the context is actually determined by no more than the $S-1$ most recent symbols.
} 
Let us define $\mathcal{P}_{S}^{*}$ as the union, over all pairs of positive integers $\left\{\left(S^{T}, S^{C}\right): S^{T}+S^{C} \leq S\right\}$, of all sets of combinations of a prefix tree with $S^{T}$ states and a suffix (context) tree with $S^{C}$ leaves. The $S$-th order context predictability of $x^{N}$, denoted $\kappa\left(x^{N}, S\right)$, is defined as the minimum fraction of errors ${ }^{3}$ achieved over $x^{N}$ among all predictors in $\mathcal{P}_{S}^{*}$.

This structure, of a transient mode followed by the context-tree mode, can be motivated by the following consideration: Note that in the transient mode, which is active at the beginning of the sequence, the predictor is actually using the entire past, $\left(x_{1}, \ldots, x_{t}\right)$, as its context. This usage of the entire past can be attributed, in a real-life situation, to "training," or "learning." During this training time, in addition to providing predictions, the system "learns," from the whole data available thus far, what are the "typical" patterns and then, on the basis of this study, it designs the context-tree predictor to be used in the context-tree mode, which will remain fixed thereafter. Since the total memory resources (given by $S$ ) are limited, they have to be divided between the training and the size of the context dictionary to be used in the context-tree mode. Thus, there is a tradeoff, but the definition of the class $\mathcal{P}_{S}^{*}$ allows the full freedom with regard to the partition between $S^{T}$ transient states and $S^{C}$ context-tree states. On the one extreme, we can take $S^{T}=0$ and $S^{C}=S$, which is a pure context-tree predictor in $\mathcal{P}_{S}$, with no transient mode at all. On the other extreme, we have $S^{T}=S-1$ and $S^{C}=1$, where resources are all devoted to the transient mode, and the context-tree has a root only, which means that the prediction $\hat{x}_{t+1}$ is constant, independently of past data.

Having defined $\mathcal{P}_{S}^{*}$, let us now allow $S$ grow with $N$, and accordingly, redefine the notation of the total number of states by $S_{N}$. For a monotonically non-decreasing sequence $\left\{S_{N}\right\}_{N \geq 1}$ of positive integers, we say that the context predictability is universally achievable w.r.t. $\left\{S_{N}\right\}_{N \geq 1}$ if there exists a randomized predictor (not necessarily a context predictor), $\hat{x}_{t}=f_{t}\left(x_{1}, \ldots, x_{t-1}\right), t=1,2, \ldots$, such that for every infinite sequence $\boldsymbol{x}=\left(x_{1}, x_{2}, \ldots\right)$

$$
\limsup _{N \rightarrow \infty}\left[\frac{1}{N} \sum_{t=1}^{N} \operatorname{Pr}\left\{\hat{x}_{t} \neq x_{t}\right\}-\kappa\left(x^{N}, S_{N}\right)\right] \leq 0,
$$

where the probabilities, $\operatorname{Pr}\left\{\hat{x}_{t} \neq x_{t}\right\}$, are w.r.t. the randomization. We say that a predictor achieves the context predictability w.r.t. $\left\{S_{N}\right\}_{N \geq 1}$ uniformly rapidly if the convergence in

\footnotetext{
${ }^{3}$ When randomized output functions are allowed, this should be redefined as the minimum expected fraction of errors, where the expectation is w.r.t. the randomization. However, it is easy to see that the best output function is always deterministic.
} 
eq. (3) is uniform, i.e.,

$$
\limsup _{N \rightarrow \infty} \max _{x^{N} \in\{0,1\}^{N}}\left[\frac{1}{N} \sum_{t=1}^{N} \operatorname{Pr}\left\{\hat{x}_{t} \neq x_{t}\right\}-\kappa\left(x^{N}, S_{N}\right)\right] \leq 0 .
$$

The questions we address are the following:

1. What is the fastest growth rate of $\left\{S_{N}\right\}$ such that the context predictability is still universally achievable w.r.t. $\left\{S_{N}\right\}_{N \geq 1}$ uniformly rapidly?

2. Whenever the context predictability is universally achievable, can we propose a (simple) universal predictor?

Theorem 1 answers both questions and tells us that this critical growth rate is linear.

Theorem 1 The context predictability w.r.t. $\left\{S_{N}\right\}_{N \geq 1}$ is universally achievable uniformly rapidly if and only if $\lim _{N \rightarrow \infty} S_{N} / N=0$.

Discussion: The proof of Theorem 1 consists of the sufficieny part, where a particular universal (horizon-dependent) predictor is proposed (Section 3) and the necessity part (Section 4). As we shall see, the universal predictor proposed in Section 3, bases its predictions on no more than $2 N / M_{N}$ contexts, where $\left\{M_{N}\right\}_{N \geq 1}$ is a sequence of positive integers tending to infinity such that $\lim _{N \rightarrow \infty} S_{N} M_{N} / N=0$, and so, the number of contexts used by the algorithm must increase slightly faster than $\left\{S_{N}\right\}$. As will be seen in Section 3, the best choice of $M_{N}$, in the sense of minimizing (the upper bound on) $\max _{x^{N} \in\{0,1\}^{N}}\left[(1 / N) \sum_{t=1}^{N} \operatorname{Pr}\left\{\hat{x}_{t} \neq x_{t}\right\}-\kappa\left(x^{N}, S_{N}\right)\right]$ is of the order of $\left(N / S_{N}\right)^{2 / 3}$, which yields a redundancy of the order of $\left(S_{N} / N\right)^{1 / 3}$. It should be noted that it is also possible to obtain a redundancy rate of $O\left(\left(S_{N} \log S_{N}\right) / N\right)$, which may be better in some cases, by using the expert-advice methodology (cf. the relevant references in [4]), where the "experts" are all the members of $\mathcal{P}_{S_{N}}^{*}$. However, the implementation of the expert-advice algorithm is extremely complex because it needs to apply all predictors of $\mathcal{P}_{S_{N}}^{*}$ in parallel. The proposed horizon-dependent algorithm is next modified to be horizon-independent.

As for the necessity part of Theorem 1 , we assume that $S_{N}=a N+1$ for some positive constant $a \leq 1$, and demonstrate that there is a set of sequences $\left\{x^{N}\right\}$ for which, on the one hand, $\kappa\left(x^{N}, a N+1\right)=0$, but on the other hand, for every universal predictor (which may be deterministic or randomized, and with unlimited resources), at least one of these 
sequences would yield no less than $a N / 2$ errors. Stated in the mathematical language, we have:

$$
\max _{x^{N} \in\{0,1\}^{N}}\left[\frac{1}{N} \sum_{t=1}^{N} \operatorname{Pr}\left\{\hat{x}_{t} \neq x_{t}\right\}-\kappa\left(x^{N}, a N+1\right)\right] \geq \frac{a}{2}
$$

for all $N$, and so, when $\lim _{N \rightarrow \infty} S_{N} / N=a>0$, the context predictability is not universally achievable uniformly rapidly. The question of universal achievability which is not uniformly rapid, in the linear case, remains open.

\section{A Universal Prediction Scheme - Proof of Sufficiency}

For a given $N$, choose a positive integer $M_{N}$, and consider the following recursive definition of prediction context, which also defines the proposed algorithm.

Let $k_{0}=k_{0}\left(x_{1}, \ldots, x_{t}\right)$ denote the largest positive integer $k$ such that the following two conditions hold at the same time:

1. The string $\left(x_{t-k+1}, \ldots, x_{t}\right)$ appears (possibly, with overlaps) at least $M_{N}$ times along $\left(x_{1}, \ldots, x_{t}\right)$.

2. The string $\left(x_{t-k+2}, \ldots, x_{t}\right)$ has already been used as the prediction context at least $M_{N}$ times in the past.

If no such $k$ exists, define $k_{0}=0$. The string $\left(x_{t-k_{0}+1}, \ldots, x_{t}\right)$ is referred to as the prediction context used at time $t$, and in the case $k_{0}=0$, the context $s_{t}$ is defined as "null," i.e., "no context."

Next, consider the prediction scheme of [1], defined w.r.t. the prediction context $s_{t}=$ $\left(x_{t-k_{0}+1}, \ldots, x_{t}\right)$. In particular, at each time instant $t$, determine the context using the above described rule, and randomly draw the prediction $\hat{x}_{t+1}$ according to the conditional distribution $p_{t}\left(\hat{x}_{t+1}=1 \mid s_{t}\right)=\phi\left(\hat{p}_{t}\left(1 \mid s_{t}\right), N\left(s_{t}\right)\right)$, where $\phi$ is defined as follows:

$$
\phi(\alpha, n)= \begin{cases}0 & \alpha<\frac{1}{2}-\epsilon_{n} \\ \frac{1}{2 \epsilon_{n}}\left(\alpha-\frac{1}{2}\right)+\frac{1}{2} & \frac{1}{2}-\epsilon_{n} \leq \alpha \leq \frac{1}{2}+\epsilon_{n} \\ 1 & \alpha>\frac{1}{2}+\epsilon_{n}\end{cases}
$$

with $\epsilon_{n} \triangleq 1 /(2 \sqrt{n+2})$, and where $\hat{p}_{t}(1 \mid s)=\left[N_{t}(s, 1)+1 / 2\right] /\left[N_{t}(s)+1\right], N_{t}(s)$ being the number of occurrences of the context $s$ (w.r.t. the above rule) along $\left(x_{1}, \ldots, x_{t-1}\right)$ and $N_{t}(s, 1)$ is the number of times these appearances of context $s$ were followed by " 1 ".

We next analyze the performance of this prediction scheme in comparison to the best reference predictor in $\mathcal{P}_{S_{N}}^{*}$, with a set $\mathcal{S}_{N}^{T}$ of $S_{N}^{T}$ transient states, and a set $\mathcal{S}_{N}^{C}$ of $S_{N}^{C}$ 
context states, $S_{N}^{T}+S_{N}^{C} \leq S_{N}$. An upper bound on the redundancy, $\left[(1 / N) \sum_{t=1}^{N} \operatorname{Pr}\left\{\hat{x}_{t} \neq\right.\right.$ $\left.\left.x_{t}\right\}-\kappa\left(x^{N}, S_{N}\right)\right]$, will be obtained by bounding $(1 / N) \sum_{t=1}^{N} \operatorname{Pr}\left\{\hat{x}_{t} \neq x_{t}\right\}$ from above, and bounding $\kappa\left(x^{N}, S_{N}\right)$ from below. We begin with the latter by counting only errors that occur during the context-tree mode of the reference predictor, which lasts at least $N-S_{N}^{T}$ time units, as the transient mode cannot last longer than $S_{N}^{T}$ instants. For the given $x^{N}$, let $\left(s_{1}, \ldots, s_{N}\right)$ be the sequence of states that would have been obtained had only the context-tree machine of the reference predictor been used, from $t=1$ to $t=N$. As is shown in [1], the number of errors made by such a (pure context-tree) predictor is given by $\sum_{s \in \mathcal{S}_{N}^{C}} \min \{N(s, 0), N(s, 1)\}$, where $N(s, x), s \in \mathcal{S}_{N}^{C}, x \in\{0,1\}$, is the number of joint occurrences of $s_{t}=s$ and $x_{t+1}=x$ along the pair of sequences $\left(s^{N}, x^{N}\right)$. The joint count of $s_{t}=s$ and $x_{t+1}=x$, during the context-tree mode only, cannot then be smaller than $N(s, x)-S_{N}^{T}$, and so,

$$
\begin{aligned}
\kappa\left(x^{N}, S_{N}\right) & \geq \frac{1}{N}\left[\sum_{s \in \mathcal{S}_{N}^{C}} \min \{N(s, 0), N(s, 1)\}-S_{N}^{T}\right] \\
& \geq \frac{1}{N}\left[\sum_{s \in \mathcal{S}_{N}^{C}} \min \{N(s, 0), N(s, 1)\}-S_{N}\right] .
\end{aligned}
$$

As was also shown in [1], when the predictor (6) is applied, the contribtution of each state $s$ to the expected number of prediction errors, $E N_{e}(s) \triangleq \sum_{t: s_{t}=s} \operatorname{Pr}\left\{\hat{x}_{t} \neq x_{t}\right\}$, is upper bounded by

$$
E N_{e}(s) \leq \min \{N(s, 0), N(s, 1)\}+\sqrt{N(s)+1}+\frac{1}{2},
$$

where $N(s)=N(s, 0)+N(s, 1)$ is the number of occurrences of $s$.

Consider the above described universal prediction scheme applied to $x^{N}$, and let us denote now the sequence of contexts, generated by this algorithm, as $\hat{s}^{N}=\left(\hat{s}_{1}, \ldots, \hat{s}_{N}\right)$ (to distinguish from the contexts of the context-tree component of the reference predictor of $\mathcal{P}_{S_{N}}^{*}$ ), and let $\hat{\mathcal{S}}_{N}$ denote the set of contexts generated this way.

We first observe that there are at most $2 M_{N} S_{N}^{C}$ times instants where $\hat{s}_{t}$ is a suffix of $s_{t} \in \mathcal{S}_{N}^{C}$. This follows from the following consideration. In a full binary tree with $S_{N}^{C}$ leaves, like the tree corresponding to the reference predictor, there are always $S_{N}^{C}-1$ internal nodes (including the root), pertaining to all possible states which are suffixes of some state in $\mathcal{S}_{N}^{C}$. Now, by construction of the algorithm, every such internal node $s^{\prime}$ is used as a prediction context no more than $2 M_{N}$ times. This is because upon the $\left(2 M_{N}+1\right)$-st time, either 
the pattern $\left(0, s^{\prime}\right)$ or $\left(1, s^{\prime}\right)$ has appeared at least $M_{N}$ times, and thus both conditions for extending the prediction context by one bit are satisfied. Thus, the total number of times that suffixes of contexts in $\mathcal{S}_{N}^{C}$ are used as prediction contexts cannot exceed $2 M_{N}\left(S_{N}^{C}-1\right)$. We will further upper bound this number by $2 M_{N} S_{N}$, for simplicity.

In the remaining time instants, of course, either $\hat{s}_{t}=s_{t}$ or $s_{t}$ becomes a suffix of $\hat{s}_{t}$. Correspondingly, for a given $s \in \mathcal{S}_{N}^{C}$, let $\mathcal{T}_{s}$ denote the sub-tree of prediction contexts, rooted at $s$, that are generated by the algorithm, i.e., all generated contexts $\{\hat{s}\}$ suffixed by $s$ (including $s$ itself as the root). Following eq. (8), the expected number of errors is bounded by

$$
\frac{1}{N} \sum_{t=1}^{N} \operatorname{Pr}\left\{\hat{x}_{t} \neq x_{t}\right\} \leq 2 M_{N} S_{N}+\sum_{s \in \mathcal{S}_{N}^{C}} \sum_{\hat{s} \in \mathcal{T}_{s}}\left[\min \{N(\hat{s}, 0), N(\hat{s}, 1)\}+\sqrt{N(\hat{s})+1}+\frac{1}{2}\right],
$$

where the first term, $2 M_{N} S_{N}$, accounts for worst case of totally erroneous prediction at all $2 M_{N} S_{N}$ visits at states $\{\hat{s}\}$ that are suffixes of some states in $\mathcal{S}_{N}^{C}$, and the second term is an upper bound on the expected number of errors at all other times. Now, let us decompose the second term into

$$
A \triangleq \sum_{s \in \mathcal{S}_{N}^{C}} \sum_{\hat{s} \in \mathcal{T}_{s}} \min \{N(\hat{s}, 0), N(\hat{s}, 1)\}
$$

and

$$
B \triangleq \sum_{s \in \mathcal{S}_{N}^{C}} \sum_{\hat{s} \in \mathcal{T}_{s}}\left[\sqrt{N(\hat{s})+1}+\frac{1}{2}\right]
$$

We shall now bound each one of them separately. As for $A$, we have

$$
\begin{aligned}
A & \leq \sum_{s \in \mathcal{S}_{N}^{C}} \min \left\{\sum_{\hat{s} \in \mathcal{T}_{s}} N(\hat{s}, 0), \sum_{\hat{s} \in \mathcal{T}_{s}} N(\hat{s}, 1)\right\} \\
& \leq \sum_{s \in \mathcal{S}_{N}^{C}} \min \{N(s, 0), N(s, 1)\} \\
& \leq N \cdot \kappa\left(x^{N}, S_{N}\right)+S_{N} .
\end{aligned}
$$

Regarding $B$, we have the following consideration: As mentioned earlier, for internal nodes in $\mathcal{T}_{s}$ (and a-fortiori for the leaves), we know that $N(\hat{s})$ cannot exceed $2 M_{N}$, and so,

$$
B \leq \sum_{s \in \mathcal{S}_{N}^{C}} \sum_{\hat{s} \in \mathcal{T}_{s}}\left(\sqrt{2 M_{N}+1}+\frac{1}{2}\right)=\left(\sqrt{2 M_{N}+1}+\frac{1}{2}\right) \cdot \sum_{s \in \mathcal{S}_{N}^{C}}\left|\mathcal{T}_{s}\right| .
$$

Now, $\sum_{s \in \mathcal{S}_{N}^{C}}\left|\mathcal{T}_{s}\right|$ is of course, upper bounded by the total number of contexts generated by the proposed universal predictor. As every internal node of the context-tree generated 
appears at least $M_{N}$ times (by the second condition that defines the algorithm), the total number of internal nodes of $\hat{\mathcal{S}}_{N}$ cannot exceed $N / M_{N}$, and so, the total number of nodes (including the leaves) cannot exceed $2 N / M_{N}+1$. Thus, $\sum_{s \in \mathcal{S}_{N}^{C}}\left|\mathcal{T}_{s}\right| \leq 2 N / M_{N}+1$, and we can further upper bound $B$ by

$$
B \leq\left(\sqrt{2 M_{N}+1}+\frac{1}{2}\right) \cdot\left(\frac{2 N}{M_{N}}+1\right),
$$

which upon normalizing by $N$ becomes

$$
\frac{B}{N} \leq\left(2 \sqrt{\frac{2}{M_{N}}+\frac{1}{M_{N}^{2}}}+\frac{1}{M_{N}}\right) \cdot\left(1+\frac{M_{N}}{2 N}\right) .
$$

The total expected excess frequency of errors (redundancy) is thus

$$
\begin{aligned}
\frac{1}{N} \sum_{t=1}^{N} \operatorname{Pr}\left\{\hat{x}_{t} \neq x_{t}\right\}-\kappa\left(x^{N}, S_{N}\right) \leq & \left(2 \sqrt{\frac{2}{M_{N}}+\frac{1}{M_{N}^{2}}}+\frac{1}{M_{N}}\right) \cdot\left(1+\frac{M_{N}}{2 N}\right)+ \\
& \frac{\left(2 M_{N}+1\right) S_{N}}{N},
\end{aligned}
$$

where the additional term comes from the first term of the r.h.s. of eq. (9) and the rightmost side of eq. (12). The conditions for vanishing redundancy are then $M_{N} \rightarrow \infty$ and $M_{N} S_{N} / N \rightarrow 0$. Both conditions can be satisfied at the same time as long as $S_{N}$ is sublinear in $N$. As the r.h.s. is independent of $x^{N}$, the convergence to zero is uniformly fast. This completes the proof of the sufficiency part.

Two comments are in order at this point:

1. Note that the asymptotically optimum growth rate of $M_{N}$ (in the sense of minimizing the r.h.s.) is $M_{N}=O\left(\left(N / S_{N}\right)^{2 / 3}\right)$, which yields $B / N \leq O\left(\left(S_{N} / N\right)^{1 / 3}\right)$.

2. The above algorithm is horizon-dependent, i.e., the length of the sequence, $N$, has to be known ahead of time in order to determine the value of $M_{N}$. It is not difficult, however, to modify this algorithm so as to be horizon-independent. One way to do that is the following: Instead of defining the required number of context repetitions, in conditions 1 and 2 of the algorithm, to depend directly on $N$, let us define it as depending on $k$, the length of the examined context. More specifically, let us replace $M_{N}$ by $M(k)$ and by $M(k-1)$ in conditions 1 and 2 , respectively, where $\{M(k)\}_{k \geq 1}$ is a certain monotonic sequence of positive integers that tends to infinity. The reader is referred to the appendix for more details on the redundancy analysis and the considerations regarding the choice of the sequence $\{M(k)\}$. It is also demonstrated, in the 
appendix, that the (upper bound on the) redundancy term of this algorithm decays faster than that of the LZ-based algorithm proposed in [1].

\section{Proof of Necessity}

Let $a \in(0,1]$ be given, and let $S_{N}=a N+1$, assuming without essential loss of generality that $a N$ is integer. Consider the recursive generation of a sequence $x^{N}$ by $x_{t}=f\left(s_{t}\right)$, $t=1,2, \ldots, N$, where $s_{t}$ is the state associated with previously generated symbols, and $f$ is the output function, corresponding to a certain member in $\mathcal{P}_{a N+1}^{*}$. Clearly, when this predictor is applied to the very same sequence that it has generated, then there are no prediction errors, and so, $\kappa\left(x^{N}, a N+1\right)=0$ for every such sequence.

Next, consider a subset of $2^{a N}$ pure transient-state predictors from $\mathcal{P}_{a N+1}^{*}$, i,e., predictors with $S_{N}^{T}=a N$ and $S_{N}^{C}=1$, whose associated $x$-sequences (generated as above) start with all $2^{a N}$ possible binary strings of length aN correspondingly. That is, the first predictor generates a sequence that begins with $a N$ zeroes, the second predictor generates a sequence whose first $a N$ bits are $(0,0, \ldots, 0,1)$, and so on. Clearly, there are enough degrees of freedom to do that: Given any desired binary string $\left(x_{1}, \ldots, x_{a N}\right)$ of the first $a N$ bits of $x^{N}$, consider the finite-state (transient) machine corresponding to a prefix tree whose internal nodes are $\emptyset$ (the null string), $\left\{x_{1}\right\},\left\{x_{1}, x_{2}\right\}, \ldots,\left\{x_{1}, \ldots, x_{a N}\right\}$, and whose leaves are $\left\{\bar{x}_{1}\right\},\left\{x_{1}, \bar{x}_{2}\right\},\left\{x_{1}, x_{2}, \bar{x}_{3}\right\}, \ldots,\left\{x_{1}, x_{2}, \ldots, x_{a N-1}, \bar{x}_{a N}\right\}, \bar{x}_{i}$ being the complement of $x_{i}, i=1, \ldots, a N$. Now, apply to each of the internal nodes an output function that will give the next desired outcome, i.e., $f(\emptyset)=x_{1}, f\left(\left\{x_{1}\right\}\right)=x_{2}$, $f\left(\left\{x_{1}, x_{2}\right\}\right)=x_{3}, \ldots, f\left(\left\{x_{1}, x_{2}, \ldots, x_{a N-1}\right\}\right)=x_{a N}$. This construction guarantees that each one of the $2^{a N}$ context-tree predictors will generate a different sequence because all these sequences differ from each other even in their first $a N$ bits.

Finally, define a random vector $X^{N}$, which is distributed uniformly across all these $2^{a N} N$-vectors. Now, for any randomized predictor, with no matter how many states, the expected fraction of errors (where the expectation is both w.r.t. the ensemble of $X^{N}$ and w.r.t. possible randomization) is lower bounded as follows:

$$
\frac{1}{N} \sum_{t=1}^{N} \operatorname{Pr}\left\{\hat{x}_{t} \neq X_{t}\right\} \geq \frac{1}{N} \sum_{t=1}^{a N} \operatorname{Pr}\left\{\hat{x}_{t} \neq X_{t}\right\}=\frac{a}{2},
$$

where the last equality is due to the fact that $\left(X_{1}, \ldots, X_{a N}\right)$ is, in fact, governed by the memoryless binary symmetric source (independent, fair coin tosses) since the distribution is 
uniform over all $2^{a N}$ strings on length $a N$. Clearly, every predictor makes exactly $50 \%$ errors on the binary symmetric source. It therefore follows that for any randomized predictor, there exists at least one vector $x^{N}$, out of the above defined ensemble of $2^{a N}$ vectors, for which the expected fraction of errors is not below $a / 2$. This completes the proof of the necessity part.

Note that for the case $a=1$, we have $\kappa\left(x^{N}, N+1\right)=0$ for every sequence, but any predictor would perform at least as bad as random guessing (50\% errors) on some sequence.

\section{Appendix}

In this appendix, we show how the performance analysis of Section 3 should be modified if the horizon-dependent algorithm is replaced by the the horizon-independent algorithm described in the second comment at the end of Section 3.

In analogy to eq. (9), we have two main redundancy terms: The first term is the summation of $2 M\left(d_{s}\right)$ over all internal nodes $\{s\}$ of the context-tree $\mathcal{S}_{N}^{C}$ (replacing the term $2 M_{N} S_{N}$ ), where $d_{s}$ stands for the depth of state $s$ in the context-tree, i.e., the distance from of $s$ from the root. This term is further bounded by $2 S_{N} \max _{s \in \mathcal{S}_{N}^{C}} M\left(d_{s}\right)=$ $2 S_{N} M\left(\max _{s \in \mathcal{S}_{N}^{C}} d_{s}\right) \leq 2 S_{N} M\left(S_{N}\right)$, where we have used the fact that the deepest leaf in a compete tree with $S_{N}$ leaves cannot be more than $S_{N}$ branches away from the root. The second term is $B$, which is now upper bounded as follows:

$$
\begin{aligned}
B & =\sum_{s \in \mathcal{S}_{N}^{C}} \sum_{\hat{s} \in \mathcal{T}_{s}}\left(\sqrt{N(\hat{s})+1}+\frac{1}{2}\right) \\
& =\sum_{s \in \mathcal{S}_{N}^{C}}\left|\mathcal{T}_{s}\right| \sum_{\hat{s} \in \mathcal{T}_{s}} \frac{1}{\left|\mathcal{T}_{s}\right|} \cdot\left(\sqrt{N(\hat{s})+1}+\frac{1}{2}\right) \\
& \leq \sum_{s \in \mathcal{S}_{N}^{C}}\left|\mathcal{T}_{s}\right|\left(\sqrt{\frac{1}{\left|\mathcal{T}_{s}\right|} \sum_{\hat{s} \in \mathcal{T}_{s}} N(\hat{s})+1}+\frac{1}{2}\right) \\
& \leq \sum_{s \in \mathcal{S}_{N}^{C}}\left|\mathcal{T}_{s}\right|\left(\sqrt{\frac{N(s)}{\left|\mathcal{T}_{s}\right|}+1}+\frac{1}{2}\right) \\
& =\sum_{s \in \mathcal{S}_{N}^{C}} \sqrt{\left|\mathcal{T}_{s}\right|} \cdot \sqrt{N^{N}(s)+\left|\mathcal{T}_{s}\right|}+\frac{1}{2} \sum_{s \in \mathcal{S}_{N}^{C}}\left|\mathcal{T}_{s}\right| \\
& \leq \sqrt{\sum_{s \in \mathcal{S}_{N}^{C}}\left|\mathcal{T}_{s}\right| \cdot \sum_{s \in \mathcal{S}_{N}^{C}}\left[N(s)+\left|\mathcal{T}_{s}\right|\right]}+\frac{1}{2} \sum_{s \in \mathcal{S}_{N}^{C}}\left|\mathcal{T}_{s}\right|
\end{aligned}
$$




$$
\leq \sqrt{\sum_{s \in \mathcal{S}_{N}^{C}}\left|\mathcal{T}_{s}\right| \cdot\left(N+\sum_{s \in \mathcal{S}_{N}^{C}}\left|\mathcal{T}_{s}\right|\right)}+\frac{1}{2} \sum_{s \in \mathcal{S}_{N}^{C}}\left|\mathcal{T}_{s}\right|
$$

where the first inequality follows from the concavity of the square-root function, and the second to the last inequality follows from the Schwartz-Cauchy inequality. Now, $\sum_{s \in \mathcal{S}_{N}^{C}}\left|\mathcal{T}_{s}\right|$, which is upper bounded by the total number of contexts generated by the algorithm, $\left|\hat{\mathcal{S}}_{N}\right|$, is in turn, upper bounded by the following consideration: Denoting by $\tilde{\mathcal{S}}_{N}$, the set of internal nodes of $\hat{\mathcal{S}}_{N}$, we have for every positive integer $j$ :

$$
\begin{aligned}
N & \geq \sum_{\hat{s} \in \tilde{\mathcal{S}}_{N}} M\left(d_{\hat{s}}\right) \\
& \geq \sum_{\hat{s} \in \tilde{\mathcal{S}}_{N}: d_{\hat{s}} \geq j} M\left(d_{\hat{s}}\right) \\
& \geq \sum_{\hat{s} \in \tilde{\mathcal{S}}_{N}: d_{\hat{s}} \geq j} M(j) \\
& \geq\left(\left|\tilde{\mathcal{S}}_{N}\right|-2^{j}+1\right) \cdot M(j),
\end{aligned}
$$

where we have used the fact that the number of nodes with depth less than $j$ cannot exceed $\sum_{i=0}^{j-1} 2^{i}=2^{j}-1$. We therefore have

$$
\left|\tilde{\mathcal{S}}_{N}\right| \leq 2^{j}-1+\frac{N}{M(j)}
$$

and so,

$$
\sum_{s \in \mathcal{S}_{N}^{C}}\left|\mathcal{T}_{s}\right| \leq\left|\hat{\mathcal{S}}_{N}\right|<2^{j+1}+\frac{2 N}{M(j)}
$$

which follows from the fact that in a complete binary tree with $m$ internal nodes, the total number of nodes is $2 m+1$. Since this is true for every $j$, we can take the minimum over $j$. Let us then denote

$$
\psi(N)=\frac{1}{N} \min _{j}\left(2^{j+1}+\frac{2 N}{M(j)}\right) \cdot=2 \min _{j}\left(\frac{2^{j}}{N}+\frac{1}{M(j)}\right) .
$$

We therefore obtain the following upper bound to the redundancy:

$$
\frac{1}{N} \sum_{t=1}^{N} \operatorname{Pr}\left\{\hat{x}_{t} \neq x_{t}\right\}-\kappa\left(x^{N}, S_{N}\right) \leq \frac{2 S_{N}\left(M\left(S_{N}\right)+1\right)}{N}+\sqrt{\psi(N)[1+\psi(N)]}+\frac{\psi(N)}{2} .
$$

The guidelines regarding the choice of the sequence $\{M(k)\}$ are, in principle, aimed at minimizing the r.h.s. of the last inequality. Obvioulsy, the faster is the growth rate of $\{M(k)\}$, the faster $\psi(N)$ decays, but on the other hand, the first term above is enlarged. Moreover, this dictates an interesting tradeoff with regard to universal achievability. If 
one wishes to compete with the context predictability for every sublinear growth rate of $\left\{S_{N}\right\}$, then $M(k)$ should be a constant $M_{0}$ (otherwise $S_{N} M\left(S_{N}\right) / N$ may not tend to zero), but then $\psi(N)$ tends to a constant, which can be made arbitrarily small for large enough $M_{0}$. Thus, the context predictability is achieved within an arbitrarily small $\epsilon>0$, but not strictly achieved. If, on the other hand, one is somewhat less ambitious, and is only interested in achieving the context predictability for slower sequences $\left\{S_{N}\right\}$, i.e., those for which $\left\{S_{N} M\left(S_{N}\right) / N\right\}$ still vanishes for a certain choice of the sequence $\{M(k)\}$, then this is accomplished by the algorithm. For example, if $M(k)=2^{k}$, then $\psi(N)=O(1 / \sqrt{N})$, but then $\left\{S_{N}\right\}$ of the reference class is only allowed to grow slower than logarithmically in $N$, for the purpose of comparison.

Finally, it is interesting to compare the performance of the proposed horizon-independent algorithm to that of the LZ-based algorithm of [1]. To this end, let us even assume that $S_{N}=S=2^{k}$ is fixed (not growing with $N$ ), and that our reference predictor is a pure context-tree algorithm (with no transient states), where the context-tree is the full binary tree whose leaves are all the $2^{k}$ binary $k$-tuples, in other words, a finite-memory ("Markov") predictor of order $k$. In [1, Theorem 4], it is asserted that the (upper bound on the) redundancy of the LZ-based predictor w.r.t. this finite-memory predictor decays at the rate of $1 / \sqrt{\log N}$. Here, on the other hand, if we choose, for example, $M(k)=2^{k}$, as suggested above, then the redundancy would decay at the rate of $N^{-1 / 4}$, which is better. Moroever, the choice $M(k)=2^{k}$ may not even be the best possible choice. One can come close to the rate of $N^{-1 / 2}$ by letting $\{M(k)\}$ grow sufficiently rapidly.

\section{References}

[1] M. Feder, N. Merhav, and M. Gutman, "Universal prediction of individual sequences," IEEE Trans. Inform. Theory, vol. 38, no. 4, pp. 1258-1270, July 1992.

[2] P. Jacquet, W. Szpankowski, and I. Apostol, "A universal predictor based on pattern matching," IEEE Trans. Inform. Theory, vol. 48, no. 6, pp. 1462-1471, June 2002.

[3] A. Martin, G. Seroussi, and M. J. Weinberger, "Linear time universal coding and time reversal of tree sources via FSM closure," IEEE Trans. Inform. Theory, vol. 50, no. 7, pp. 1442-1468, July 2004. 
[4] N. Merhav and M. Feder, "Universal prediction," IEEE Trans. Inform. Theory, vol. 44, no. 6, pp. 2124-2147, October 1998. Also, in Information Theory: 50 Years of Discovery, pp. 80-103, Eds. S. Verdú and S. McLaughlin, IEEE Press, 1999.

[5] Y. M. Shtarkov, T. J. Tjalkens, and F. M. J. Willems, "Multialphabet weighting universal coding of context tree sources," Problems of Information Transmission (IPPI), vol. 33 , no. 1 , pp. 17-28, 1997.

[6] M. J. Weinberger and G. Seroussi, "Sequential prediction and ranking in universal context modeling and data compression," HPL Technical Report no. HPL-94-111, November 1994.

[7] M. J. Weinberger, G. Seroussi and G. Sapiro, "LOCO**2I: A low complexity, contextbased, lossless image compression algorithm," Proc. DCC ‘96, Snowbird, Utah, March 1996.

[8] F. M. J. Willems, "The context-tree weighting method: extensions," IEEE Trans. Inform. Theory, vol. 50, no. 7, pp. 1442-1468, July 2004.

[9] F. M. J. Willems, Y. M. Shtar'kov, and T. J. Tjalkens, "The context-tree weighting method: basic properties," IEEE Transactions on Information Theory, vol. 44, no. 2, pp. 792-798, March 1998.

[10] J. Ziv and A. Lempel, "Compression of individual sequences via variable-rate coding," IEEE Trans. Inform. Theory, vol. IT-24, no. 5, pp. 530-536, September 1978.

[11] J. Ziv, "An efficient universal prediction algorithm for unknown sources with limited training data," IEEE Trans. Inform. Theory, vol. 48, no. 6, pp. 1690-1693, June 2002.

[12] J. Ziv, "Correction to: "An efficient universal prediction algorithm for unknown sources with limited training data' [1]," IEEE Trans. Inform. Theory, vol. 50, no. 8, pp. 1851-1852, August 2004. 